\title{
Effects of beetroot juice on daily and 24-hour ambulatory blood pressure monitoring in older overweight and obese subjects
}

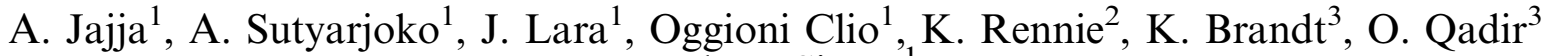 \\ and M. Siervo 1 \\ ${ }^{1}$ Institute for Ageing and Health, Newcastle University, Newcastle Upon Tyne, UK, ${ }^{2}$ School of Life and Medical \\ Sciences, University of Hertfordshire, Hatfield, UK and ${ }^{3}$ School of Agriculture, Food and Rural Development \\ Newcastle University, Newcastle Upon Tyne, UK
}

Background: Inorganic nitrate supplementation has been associated with decreased systolic blood pressure (BP). However, these findings come from short-term studies on mostly healthy young subjects (1). We studied the effects of beetroot juice supplementation on BP in overweight and obese older participants and we evaluated whether changes in BP were sustained after the interruption of the supplementation.

Methods and Results: Twenty-four participants were randomised to either beetroot or blackcurrant juice supplementation for 3 weeks. After the third week, supplementation was stopped in both groups and participants returned for their final visit after 7 days (week 4 , carry-over phase). Changes in systolic and diastolic BP were measured during the supplementation and carry-over phases. BP was measured using three different methods: 1) resting clinic BP; 2) 24-hr ambulatory BP monitoring (ABMP) and 3) home monitoring of daily resting BP. The first two methods were applied at baseline, week 3 and week 4 whereas daily measurements were performed in duplicate throughout the study by each participant in the morning and at night (method 3). A linear mixed model adjusted for baseline values was used to assess differences in BP responses between the two interventions.

Twenty-one subjects completed the study (Beetroot/Blackcurrant $=10 / 11$; Male/Female $=12 / 9 ;$ Age $=62 \cdot 0 \mathrm{SD} 1 \cdot 4 \mathrm{years} ; \mathrm{BMI}=30 \cdot 1$ SD $1.2 \mathrm{~kg} / \mathrm{m}^{2}$ ). The two groups were matched for baseline anthropometric and BP measurements. Beetroot juice supplementation was not associated with significant changes in resting clinic BP and 24-hr ABPM (Figure 1, left panel) in both phases (supplementation, recovery). A significant effect of beetroot juice on daily systolic BP was observed after 3 weeks $(-7.3 \pm 5.9 \mathrm{mmHg}, p=0.02)$ but the effect was not maintained after the interruption of the supplementation (week $4,-2 \cdot 8 \pm 6.1 \mathrm{mmHg}, p=0.09)($ Figure 1 , right panel).
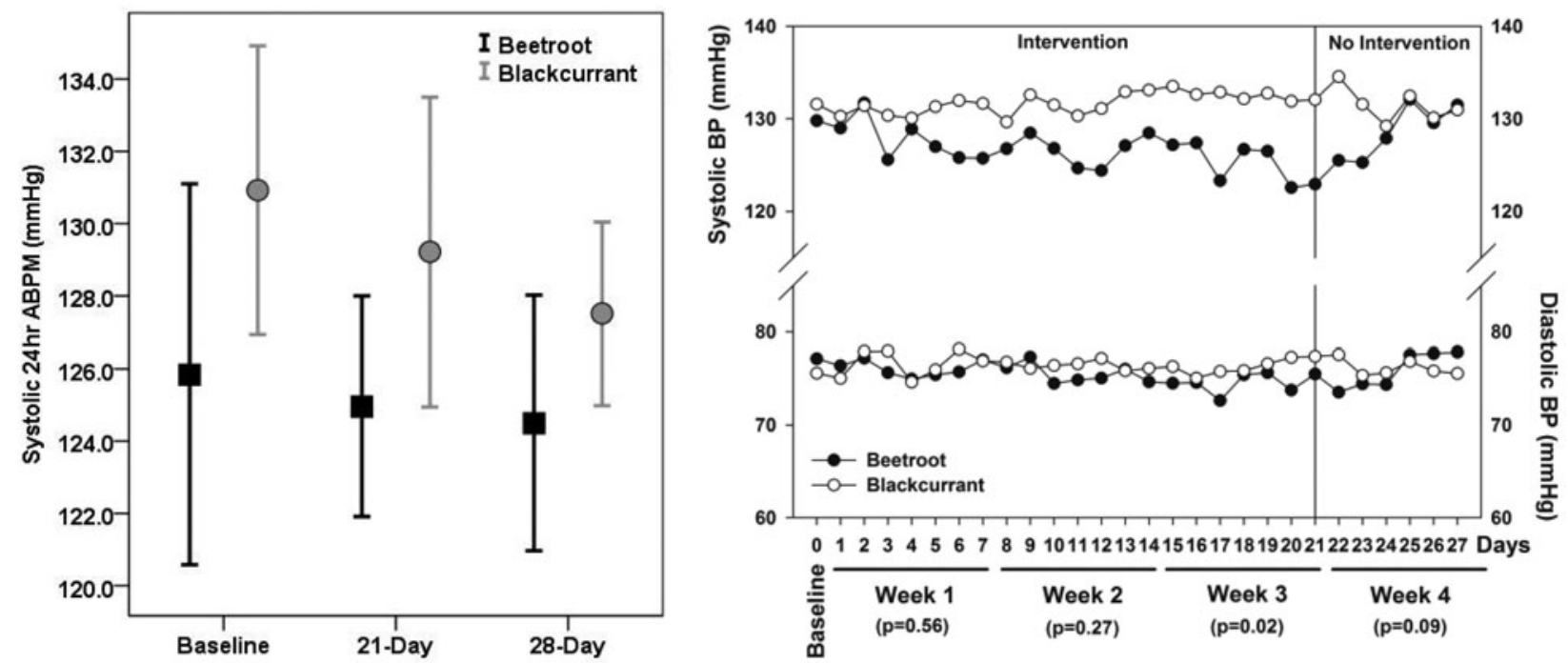

Fig. 1. Mean changes in systolic 24-hr ABPM (left panel) and daily systolic and diastolic BP (right panel) in subjects randomised to beetroot and blackcurrant juice. Error bars are SEM.

Conclusions: The effect of beetroot juice supplementation in older overweight subjects appeared to be influenced by the method used to measure BP. The daily monitoring of BP could represent a sensitive method to evaluate the efficacy of nutritional interventions on BP.

1. Siervo M, et al. (2013) Inorganic nitrate and beetroot juice supplementation reduces blood pressure in adults: a systematic review and meta-analysis. J Nutr. 143, 818-26. 